\title{
MAGNETOHYDRODYNAMIC SHOCKS AND SOLITONS IN THE SOLAR ATMOSPHERE: RECENT CHALLENGES IN OBSERVATIONS AND THEORY
}

\author{
T. V. Zaqarashvili*, K. Murawski ${ }^{\dagger}$, M. L. Khodachenko*, \\ V. Kukhianidze ${ }^{\ddagger}$, and H. O. Rucker*
}

\begin{abstract}
Magnetohydrodynamic shocks are believed to play a significant role in the dynamics of lower solar atmosphere. Here we review the recent theoretical developments in shock wave induced phenomena and first observational evidence of slow sausage soliton in the solar chromosphere.
\end{abstract}

\section{Introduction}

Pulse or wave trains generated in the photosphere and propagating upwards are quickly developed into shocks due to the rapid decrease of plasma density with height. The shocks lead to the occurrence of spicules: jets of cold chromospheric plasma flowing upwards into the hot corona. Spicules may provide the solar corona with mass, which is lost due to the solar wind. Many properties of spicules are still unexplained, for example, the double thread structure and bi-directional flows [Tanaka, 1974; Tsiropoula, 1994; Suematsu et al., 2008]. The observed oscillations of spicule axis may reveal the process of energy transport through the chromosphere [Zaqarashvili et al., 2007; Zaqarashvili and Erdélyi, 2009]. On the other hand, the shock waves propagating along magnetic flux tubes may lead to the formation of soliton, stable structure moving without significant change in shape and speed. Recent observational data with unprecedentedly high spatial resolution obtained by Solar Optical Telescope (SOT) on board of Hinode spacecraft revealed the first observational evidence of soliton in the solar atmosphere [Zaqarashvili et al., 2010]. Here we briefly review the observation of soliton and recent development in the modeling of spicule formation.

\footnotetext{
* Space Research Institute, Austrian Academy of Sciences, Schmiedlstrasse 6, A-8042 Graz, Austria

$\dagger$ Group of Astrophysics, UMCS, ul. Radziszewskiego 10, 20-031, Lublin, Poland

$\ddagger$ Abstumani Astrophysical Observatory, Ilia State University, Kazbegi ave. 2a, Tbilisi, Georgia
} 


\section{First Observation of Slow Sausage Soliton in the Solar Atmo- sphere}

We use Ca II H time series of quiet Sun regions observed by Hinode/SOT (Fig.1, upper panel). The observational sequence run on 22th November, 2006 from 05:57:31 U.T. to 06:34:57 U.T. The exposure time for each image is $0.512 \mathrm{~s}$. The integration time for each step of time series is uniform and equal to $4.8 \mathrm{~s}$.

The time series between 06:19:01 and 06:19:36 U.T. clearly shows upward propagating pattern in the form of intensity blob (Fig. 1, lower panel). The blob appears at 500-600 $\mathrm{km}$ height above the surface and reaches to the height of $\sim 1700 \mathrm{~km}$ after $35 \mathrm{~s}$ [Zaqarashvili et al. 2010]. Therefore, the mean apparent propagation speed is

$$
35 \mathrm{~km} \mathrm{~s}^{-1} \text {. }
$$

The blob has elongated form and the length to width ratio is $\sim 3$ in average.

Analytical solution of the sausage soliton in a magnetic slab is [Roberts and Mangeney, 1982; Ruderman, 2003]

$$
\eta=\frac{a l^{2}}{l^{2}+[z-s t]^{2}}
$$

where $\eta$ is the displacement of the slab boundary, $a$ is the maximal value of the displacement $\eta$ (i.e. the soliton amplitude) and

$$
s=c_{T}+\frac{1}{4} \frac{a b}{x_{0}}, l=4 \frac{\kappa x_{0}}{a b}
$$

are the soliton speed and the spatial scale respectively.

The parameters $b$ and $\kappa$ are expressed as

$$
b=\frac{V_{A}^{4}\left[3 c_{s}^{2}+(\gamma+1) V_{A}^{2}\right]}{2 c_{T}\left(c_{s}^{2}+V_{A}^{2}\right)^{2}}, \kappa=\frac{x_{0}}{2} \frac{\rho_{e 0}}{\rho_{0}} \frac{c_{T} c_{s}^{2}\left(c_{T}^{2}-V_{A e}^{2}\right)}{m_{e} V_{A}^{2}\left(c_{s}^{2}+V_{A}^{2}\right)},
$$

where $V_{A}\left(V_{A e}\right)$ is the Alfvén speed inside (outside) the slab, $c_{s}$ is the sound speed, $c_{T}$ is the tube speed, $x_{0}$ is the slab half width, $\rho_{0}\left(\rho_{e 0}\right)$ is the density inside (outside) the slab, $\gamma$ is the ratio of specific heats, $m_{e}=\sqrt{\left(V_{A e}^{2}-c_{T}^{2}\right)\left(c_{s e}^{2}-c_{T}^{2}\right) /\left[\left(V_{A e}^{2}+c_{s e}^{2}\right)\left(c_{T e}^{2}-c_{T}^{2}\right)\right]}$ is the perpendicular wave number outside the slab.

Observed intensity blob fits to a slow sausage soliton in chromospheric magnetic tube, which has the Alfvén speed of $\sim 70 \mathrm{~km} \mathrm{~s}^{-1}$ and the temperature balance with surroundings [Zaqarashvili et al. 2010].

\section{Shock Waves as Important Part of Spicule Formation Mech- anism in the Solar Atmosphere}

Spicules are chromospheric cold plasma jets raising into the hot corona with average speed of 20-25 $\mathrm{km} \mathrm{s}^{-1}$. They are usually detected in chromospheric $\mathrm{H} \alpha, \mathrm{D}_{3}$ and Ca II H 

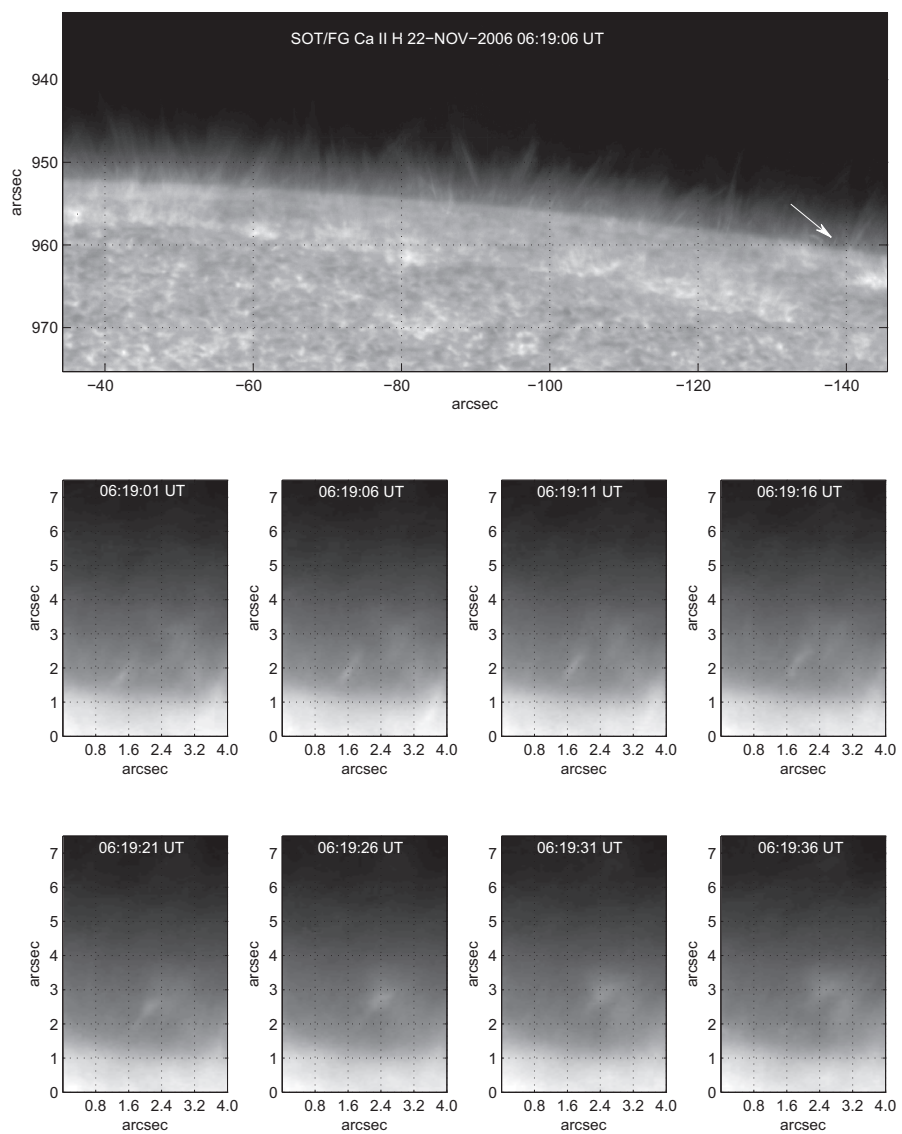

Figure 1: Upper panel: Ca II H image of quiet Sun obtained by Hinode/SOT, where the white arrow shows the place of intensity blob propagation. Lower panel: 8 consecutive images of time sequence in Ca II H line (left to right and top to bottom). The intensity blob is located near $(x, y)=(1.4,1.8)$ point in the first image. The blob propagates upward with mean apparent speed of $35 \mathrm{~km} \mathrm{~s}^{-1}$. We identify it with the slow sausage soliton propagating along magnetic flux tube. The plots are adopted from Zaqarashvili et al. [2010].

lines as thin and elongated structures. They are much denser than surrounding coronal plasma. Most spicules show up a double thread structure and bi-directional flow during their evolution [Tanaka, 1974; Tsiropoula, 1994; Suematsu et al., 2008]. They also show quasi periodic up and down motion with period of 5-min.

There are several energy sources which can be responsible for the formation of spicules: photospheric convection (granulation), photospheric oscillations (p-modes), explosive events 

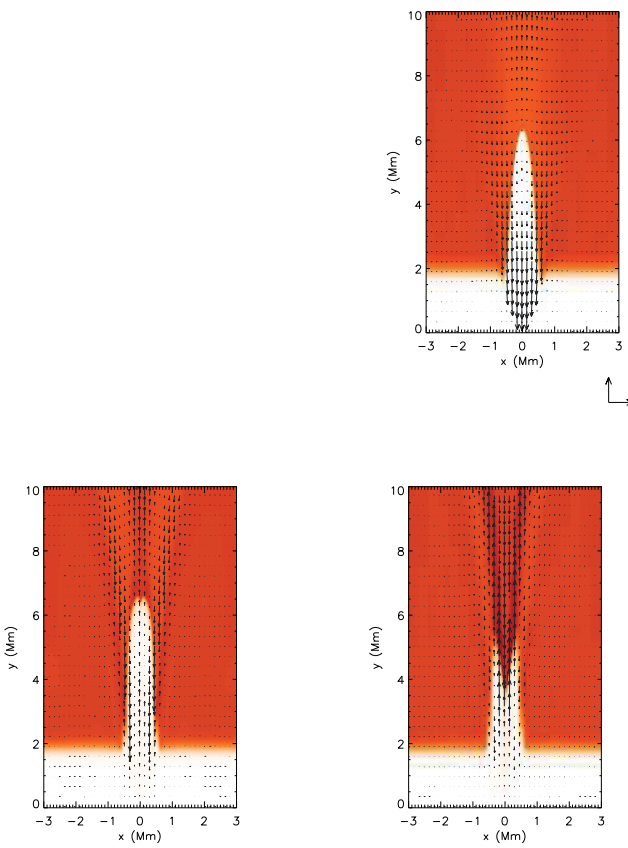

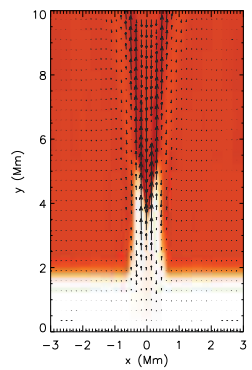

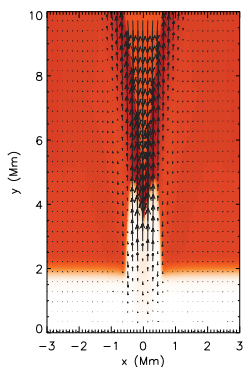

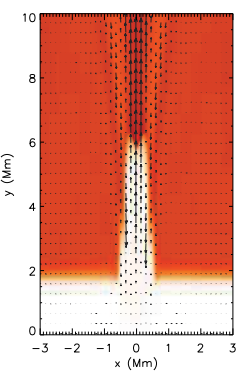

Figure 2: Temperature (contour plots) and velocity (arrows) profiles at $t=50 \mathrm{~s}, t=190 \mathrm{~s}, t=360$ $\mathrm{s}, t=500 \mathrm{~s}, t=790 \mathrm{~s}$, and $t=890 \mathrm{~s}$ for the initial amplitude of $30 \mathrm{~km} \mathrm{~s}^{-1}$. The plots show the quasi-periodic rise of spicules in the solar corona with mean period of $5 \mathrm{~min}$. This fits well with spectroscopic and imaging observations. Temperature is drawn in units of $1 \mathrm{MK}$. The arrow below each panel represents the length of the velocity vector, expressed in units of $1 \mathrm{Mm} \mathrm{s}^{-1}$. The colour bar is common to all panels. The plots are adopted from Murawski and Zaqarashvili [2010].

(magnetic reconnection) etc. Granulation and reconnection can be approximated as velocity/pressure pulses launched in the lower atmosphere, while the photospheric oscillations can be approximated by quasi-periodic drivers. In order to study the spicule formation process we solve the full set of 2-dimensional magnetohydrodynamic equations numerically with the code FLASH. We use the atmospheric model with the realistic stratification of density and the smoothed step function profile of temperature, where the temperature is raised from $1.5 \times 10^{4} \mathrm{~K}$ to $3 \mathrm{MK}$ over $200 \mathrm{~km}$. Here we use the impulsive driver and launch initial velocity pulse at the chromospheric base in order to study its tempo-spatial evolution [Murawski and Zaqarashvili, 2010].

Figure 2 displays the spatial profiles of vertical velocity (arrows) and plasma temperature 
resulting from the initial velocity pulse that was launched at the point $\left(x_{0}=0, y_{0}=0.5\right)$ $\mathrm{Mm}$, at which the magnetic field is essentially vertical. The upper left panel corresponds to $t=50 \mathrm{~s}$. Chromospheric plasma lags behind the shock front and reaches the level of $\mathrm{y}=3.0 \mathrm{Mm}$. The reason of the material being lifted up is the rarefaction of the plasma behind the shock front, which leads to under pressure there. As a result the pressure gradient force works against gravity and it forces the chromospheric material to penetrate the solar corona. The next snapshot corresponds to $t=190 \mathrm{~s}$, when the chromospheric plasma already reached the level of $y=6.5 \mathrm{Mm}$. The structure clearly resembles a spiculelike structure with the chromospheric temperature. The density of the structure is also close to the chromospheric density (not shown in the figure). Its width is

$$
600-700 \mathrm{~km}
$$

and mean rising speed is

$$
25 \mathrm{~km} \mathrm{~s}^{-1} \text {. }
$$

These values, which are obtained from the initial pulse with typical photospheric parameters, are close to the corresponding characteristics of spicules. At $t=190 \mathrm{~s}$ the plasma already flows downwards being attracted by gravity.

In following panels we see how the plasma moves up and down showing double and sometimes triple structure. The up and down motion occurs with period of

$$
\sim 5 \min .
$$

This periodicity is produced due to the nonlinear wake, which forms after propagation of pulse in the stratified atmosphere. The wake leads to the consecutive shocks (called as rebound shocks by Hollweg [1982]). The consecutive shocks force chromospheric plasma to quasi-periodic rise up into the corona.

\section{Results and Conclusions}

Hinode/SOT observations in Ca II H line show the propagation of intensity blob in the solar atmosphere. The observed properties of the blob correspond to the slow surface sausage soliton propagating with $35 \mathrm{~km} \mathrm{~s}^{-1}$ speed in the magnetic flux tube, which has the Alfvén speed of $\sim 70 \mathrm{~km} \mathrm{~s}^{-1}$ and is in the temperature balance with surroundings. This is the first observational evidence of soliton in the solar atmosphere.

The consecutive shocks, which are formed due to the nonlinear wake behind the propagating pulse in the stratified atmosphere, lead to quasi periodic rise of the chromospheric plasma into the corona and thus resembles the dynamics of spicules. The period between consecutive pulses is $\sim 5 \mathrm{~min}$, which fits with observations.

The interaction of up and down motions leads to the occurrence of double (sometimes even triple) structures during the evolution of spicules. Our results give the first reliable explanation of double structures and bidirectional flows frequently observed in spicules. 
Acknowledgments. This work was supported by the Austrian Fonds zur Förderung der wissenschaftlichen Forschung (project P21197-N16) and the Georgian National Science Foundation grant GNSF/ST09/4-310. Hinode is a Japanese mission developed and launched by ISAS/JAXA, with NAOJ as domestic partner and NASA and STFC (UK) as international partners. It is operated by these agencies in co-operation with ESA and NSC (Norway).

\section{References}

Hollweg, J. V., On the origin of solar spicules, Astrophys. J., 257, 345-353, 1982.

Murawski, K. and T. V. Zaqarashvili, Numerical simulations of spicule formation in the solar atmosphere, Astron. Astrophys., 474, A8, 2010.

Roberts, B. and A. Mangeney, Solitons in solar magnetic flux tubes, Month. Not. R. Astron. S., 198, 7-11, 1982.

Ruderman, M. S., Nonlinear waves in magnetically structured solar atmosphere, in Turbulence, Waves, and Instabilities in the Solar Plasma edited by R. Erdélyi, et al., Kluwer Academic Publisher, Dordrecht, 239-265, 2003.

Suematsu, Y., K. Ichimoto, Y. Katsukawa, T. Shimizu, T. Okamoto, S. Tsuneta, T. Tarbell, and R. A. Shine, High Resolution Observations of Spicules with Hinode/SOT, ASP Conference Series, 397, 27-31, 2008.

Tanaka, K., Evolution of Chromospheric Fine Structures on the Disk, in Chromospheric Fine Structure, edited by G. Athay, IAU Symp., 56, 239-255, 1974.

Tsiropoula, G., C.E. Alissandrakis and B. Schmieder, Time evolution of fine structures in the solar chromosphere, Astron. Astrophys., 290, 285-294, 1994.

Zaqarashvili, T. V. and R. Erdélyi, Oscillations and Waves in Solar Spicules, Space Sci. Rev., 149, 355-388, 2009.

Zaqarashvili, T.V., E. Khutsishvili, V. Kukhianidze and G. Ramishvili, Doppler-shift oscillations in solar spicules, Astron. Astrophys., 519, 627-632, 2007.

Zaqarashvili, T. V., V. Kukhianidze, and M. S. Khodachenko, Propagation of a sausage soliton in the solar lower atmosphere observed by Hinode/SOT, Month. Not. $R$. Astron. S., 404, L74-L78, 2010. 